\title{
Better Approximation Bounds for the Joint Replenishment Problem*
}

\author{
Marcin Bienkowski ${ }^{1}$, Jaroslaw Byrka ${ }^{1}$, Marek Chrobak $^{2}$, Łukasz Jeż ${ }^{1,3}$ and Jiří Sgall ${ }^{4}$ \\ ${ }^{1}$ Institute of Computer Science, University of Wrocław, Poland. \\ ${ }^{2}$ Department of Computer Science, University of California at Riverside, USA. \\ ${ }^{3}$ Department of Computer, Control, and Management Engineering, Sapienza University \\ of Rome, Italy. \\ ${ }^{4}$ Computer Science Institute, Faculty of Mathematics and Physics, Charles University, \\ Czech Republic.
}

\begin{abstract}
The Joint Replenishment Problem (JRP) deals with optimizing shipments of goods from a supplier to retailers through a shared warehouse. Each shipment involves transporting goods from the supplier to the warehouse, at a fixed cost C, followed by a redistribution of these goods from the warehouse to the retailers that ordered them, where transporting goods to a retailer $\rho$ has a fixed cost $\mathrm{c}_{\rho}$. In addition, retailers incur waiting costs for each order. The objective is to minimize the overall cost of satisfying all orders, namely the sum of all shipping and waiting costs.

JRP has been well studied in Operations Research and, more recently, in the area of approximation algorithms. For arbitrary waiting cost functions, the best known approximation ratio is 1.8 . This ratio can be reduced to $\approx 1.574$ for the JRP-D model, where there is no cost for waiting but orders have deadlines. As for hardness results, it is known that the problem is $\mathbb{A P X}$-hard and that the natural linear program for JRP has integrality gap at least 1.245. Both results hold even for JRP-D. In the online scenario, the best lower and upper bounds on the competitive ratio are 2.64 and 3, respectively. The lower bound of 2.64 applies even to the restricted version of JRP, denoted JRP-L, where the waiting cost function is linear.

We provide several new approximation results for JRP. In the offline case, we give an algorithm with ratio $\approx 1.791$, breaking the barrier of 1.8 . In the online case, we show a lower bound of $\approx 2.754$ on the competitive ratio for JRP-L (and thus JRP as well), improving the previous bound of 2.64. We also study the online version of JRP-D, for which we prove that the optimal competitive ratio is 2 .
\end{abstract}

${ }^{*}$ Research partially supported by NSF grants CCF-1217314 and OISE-1157129, MNiSW grant no. N N206 368839, 2010-2013, EU ERC project 259515 PAAl, CE-ITI (project P202/12/G061 of GA ČR), and grant IAA100190902 of GA AV ČR. 


\section{Introduction}

The Joint Replenishment Problem (JRP) deals with optimizing shipments of goods from a supplier to a set $\mathcal{R}$ of retailers through a shared warehouse. Over time, retailers issue orders for items. All ordered items must be subsequently shipped to the retailers, although some shipments can be delayed, in order to aggregate orders into fewer shipments to reduce cost.

Specifically, for each $\rho \in \mathcal{R}$ we are given the cost $\mathrm{c}_{\rho}$ of transporting goods from the warehouse to $\rho$. We are also given the cost $C$ of transporting goods from the supplier to the warehouse. A shipment of goods from the supplier to a subset $S \subseteq \mathcal{R}$ of retailers involves first shipping them to the warehouse and then redistributing them to all retailers in $S$, at cost equal $\mathrm{C}+\sum_{\rho \in S} \mathrm{c} \rho$. Note that this cost is independent of the set of items shipped. The waiting cost of an item $\pi$ ordered at time $a$ and delivered at time $t \geq a$ is given by a function $h(t)$, possibly dependent on $\pi$, where we assume that the values of $h(t)$ are non-decreasing with $t$. The objective is to minimize the overall cost of satisfying all orders, namely the total cost of shipments plus the total waiting cost of all orders.

There are two natural restrictions on waiting costs that have been previously considered in the literature. One is to assume that the waiting costs are linear, that is $h_{\pi}(t)=t-a_{\pi}$, where $a_{\pi}$ is the arrival time of an order $\pi$. We denote this version by JRP-L. In the other version, called JRP with deadlines (JRP-D), there is no waiting cost but ordered items must be shipped before pre-specified deadlines.

Several different, but mathematically equivalent, definitions of JRP can be found in the literature. In earlier papers JRP is phrased as an inventory management problem, where the inventory of some commodity needs to meet a set of demands that arrive over time. The objective is to balance the cost of order ${ }^{1}$ that replenish the inventory with the cost of maintaining it (the so-called holding cost). This formulation would not quite make sense in the online scenario, since the orders that need to be scheduled take place before demands. An online model of JRP, referred to as Make-to-Order JRP, was introduced by Buchbinder et al. [6]. In their description there is no inventory; instead, a collection of demands must be satisfied by subsequent orders. Except for minor terminology variations, our definition is essentially the same as that in [6]. Some of recent papers [2, 5, 7, 4] on control message aggregation in networks, introduce a model where control packets (corresponding to orders, in our definition) need to be transmitted to a common destination (corresponding to the supplier), paying the transmission and delay costs. In particular, the flat-tree case studied in [5] is equivalent to JRP-L.

JRP has been well studied in Operations Research and, more recently, in the area of approximation algorithms. The problem is known to be strongly $\mathbb{N P}$-hard, even for the special cases of JRP-D and JRP-L [1, 2, 12]. APX-hardness proofs, even for some restricted versions of JRP-D, were given by Nonner and Souza [12] and Bienkowski et al. 3]. The first approximation algorithm, with ratio 2, was provided by Levi, Roundy and Shmoys [9], and was subsequently improved by Levi et al. [10, 11] to 1.8 (see also [8]). For JRP-D, the ratio was reduced to 5/3 by Nonner and Souza [12] and then to $\approx 1.574$ by Bienkowski et al. [3]. All upper bounds are based on randomized rounding of the natural linear program for JRP. As shown in [3, the integrality gap of this linear program is at least 1.245, even for JRP-D.

The online version of JRP was studied in the earlier discussed paper by Buchbinder et al. [6], who give a 3-competitive algorithm, using a primal-dual scheme, and show a lower bound of 2.64 on the competitive ratio, even for JRP-L. (See also Brito et al. [5] for related work.)

Our contributions. We provide several new approximation results for JRP. In the offline case, we give an algorithm with approximation ratio $\approx 1.791$, breaking the barrier of 1.8 from [10, 11. The improvement is achieved by refining the analysis of the LP-rounding algorithm in [10, 11] and combining it with a new algorithm that uses an approximation for JRP-D from [3].

We also study online algorithms for JRP. We show that deterministic online algorithms, even

\footnotetext{
${ }^{1}$ Note that the meaning of the term "order" here is different from our usage.
} 
for JRP-L, cannot be better than $\approx 2.754$-competitive, improving the bound of 2.64 from $[6$. For JRP-D, we prove that the optimal competitive ratio is 2 .

For convenience, we use a model where time is continuous, while some of previous works on this topic used the discrete-time model. Algorithms for the continuous model can be easily translated into the discrete model, preserving the same performance guarantee. In our lower bound proofs all waiting-cost functions are left-continuous, and for such functions lower bound arguments for competitive ratios carry over to the discrete case as well. This relationship will be formally spelled out in the final version of this paper (see a similar argument in [6]).

\section{Preliminaries}

We now review our terminology and formalize the definition of JRP. Recall that $\mathcal{R}$ denotes the set of retailers. Each order can be specified by a triple $\pi=(\rho, a, h)$, where $a$ is the time when $\pi$ was issued, $\rho$ is the retailer that issued $\pi$, and $h()$ is the waiting cost function of $\pi$, where $h(t)=\infty$ for $t<a$ and $h(t)$ is non-decreasing for $t \geq a$. Let $\Pi$ be the set of all orders. In JRP-L we will assume that $h(t)=t-a$ for $t \geq a$, and in JRP-D we have $h(t)=0$ for $a \leq t \leq d$ and $h(t)=\infty$ otherwise. Then $d$ is called the deadline of order $\pi$. In JRP-D we will in fact specify an order by a triple $\pi=(\rho, a, d)$.

A shipment is specified by a pair $(S, t)$, where $S$ is the set of retailers receiving the shipment and $t$ is the time of the shipment. The cost of shipment $(S, t)$ is $\mathrm{C}+\sum_{\rho \in S} \mathrm{c}_{\rho}$. A schedule is a set $\bar{S}$ of shipments. An order $\pi=(\rho, a, h)$ is said to be pending in $\bar{S}$ at time $\tau$ if $a \leq \tau$ and there is no shipment $(S, t)$ in $\bar{S}$ with $\rho \in S$ and $a \leq t<\tau$. If $\pi=(\rho, a, h)$ is pending at time $t$ and $(S, t)$ is a shipment in $\bar{S}$ such that $\rho \in S$, then we say that $(S, t)$ satisfies $\pi$. In such case, the waiting cost of $\pi$ in $\bar{S}$ is $h(t)$. The cost of $\bar{S}$ is the sum of its shipment and waiting costs, that is $\operatorname{Cost}(\bar{S})=\operatorname{CosT}_{\mathrm{SHIP}}(\bar{S})+\operatorname{CosT}_{\mathrm{WAIT}}(\bar{S})$, where

$$
\operatorname{CosT}_{\mathrm{SHIP}}(\bar{S})=\sum_{(S, t) \in \bar{S}}\left(\mathrm{C}+\sum_{\rho \in S} \mathrm{c}_{\rho}\right) \quad \text { and } \quad \operatorname{CosT}_{\mathrm{WAIT}}(\bar{S})=\sum_{\pi=(\rho, a, h) \in \Pi} \min _{\substack{(S, t) \in \bar{S} \\ \rho \in t \geq a}} h(t) \text {, }
$$

where $\min \emptyset \equiv+\infty$. The objective of JRP is to compute a schedule $\bar{S}$ with minimum $\operatorname{CosT}(\bar{S})$.

We use the standard definition of approximation algorithms. We will say that a polynomialtime algorithm $\mathcal{A}$ is an $R$-approximation algorithm for JRP if for any instance it computes a schedule of shipments whose cost is at most $R$ times the optimal cost for this instance.

In the online scenario, orders arrive over time, and at each time $t$ an online algorithm must decide whether to ship at time $t$ and, if so, to which retailers, based only on the existing orders. For online algorithms we use the term " $R$-competitive" as a synonym of " $R$-approximation".

In the literature, some authors distinguish between absolute approximation ratios (as defined above) and asymptotic ratios, where an algorithm is allowed to pay some additional constant overhead cost, independent of the instance. While our upper bounds apply to the absolute ratio, our lower bound proofs can be extended to the asymptotic ratios by repeating the lower bound strategies a sufficient number of times.

\section{An Upper Bound of 1.791 for Offline JRP}

We now present our 1.791-approximation algorithm. The algorithm first computes an optimal solution $\left(x^{*}, y^{*}\right)$ of the linear program for JRP. Then it chooses randomly one of three different LP-rounding methods, with probabilities and other parameters suitably optimized, to obtain a ratio improving the bound of 1.8 from [10, 11].

Linear program. Let $T=\{a:(\rho, a, h) \in \Pi\}$ be the times when orders are placed. We can assume that all shipments occur at times in $T$. We use the following indicator variables: $x_{a}$ 
represents a supplier-to-warehouse shipment at time $a, x_{\rho, a}$ represents a warehouse-to-retailer $\rho$ shipment at time $a$, and $y_{\pi, a}$ represents an order $\pi$ being satisfied by a shipment at time $a$. The following linear program is the fractional relaxation of the natural integer program for JRP.

$$
\begin{aligned}
& \text { minimize } \quad \sum_{a \in T} \mathrm{C} \cdot x_{a}+\sum_{a \in T} \sum_{\rho \in \mathcal{R}} \mathrm{c}_{\rho} \cdot x_{\rho, a}+\sum_{\pi=(\rho, a, h) \in \Pi} \sum_{t \in T: t \geq a} h(t) \cdot y_{\pi, t} \\
& \text { subject to } \quad x_{a} \geq x_{\rho, a} \quad \text { for all } a \in T, \rho \in \mathcal{R} \\
& x_{\rho, a} \geq y_{\pi, a} \quad \text { for all } \pi=(\rho, a, h) \in \Pi \\
& \sum_{t \geq a} y_{\pi, t} \geq 1 \quad \text { for all } \pi=(\rho, a, h) \in \Pi \\
& x_{a}, x_{\rho, a}, y_{\pi, a} \geq 0 \quad \text { for all } a \in T, \rho \in \mathcal{R}, \pi \in \Pi
\end{aligned}
$$

Throughout the rest of the paper, we will fix an optimal (fractional) solution to the LP above and denote it by $\left(x^{*}, y^{*}\right)$. Note that constraints (3) are satisfied with equality in $\left(x^{*}, y^{*}\right)$.

Algorithms 2SRP and 1SRP. The cost of any solution $(x, y)$ to the LP above can be naturally split into three parts: the supplier-to-warehouse shipping cost, $\operatorname{CosT}_{\mathrm{WSH}}(x, y)$; the warehouseto-retailers shipping cost, $\operatorname{CosT}_{\mathrm{RSHIP}}(x, y)$; and the waiting cost, $\operatorname{CosT}_{\mathrm{WAIT}}(x, y)$. When the solution $(x, y)$ is a random variable, these denote appropriate expected costs. We say that a solution $(x, y)$ is an $\left(r_{1}, r_{2}, r_{3}\right)$-approximation of $\left(x^{*}, y^{*}\right)$ if the following three conditions hold:

- $\operatorname{CosT}_{\mathrm{WSHIP}}(x, y) \leq r_{1} \cdot \operatorname{CosT}_{\mathrm{WSHIP}}\left(x^{*}, y^{*}\right)$,

- $\operatorname{Cost}_{\mathrm{RSHIP}}(x, y) \leq r_{2} \cdot \operatorname{Cost}_{\mathrm{RSHIP}}\left(x^{*}, y^{*}\right)$, and

- $\operatorname{CosT}_{\mathrm{WAIT}}(x, y) \leq r_{3} \cdot \operatorname{CosT}_{\mathrm{WAIT}}\left(x^{*}, y^{*}\right)$.

In our solution, we build on two LP-based, polynomial-time algorithms of Levi et al. [10]. Both are based on random shifting. The first one (denoted 2SRP) is called Two-Sided Retailer Push Algorithm the second one (denoted 1SRP) is called One-Sided Retailer Push Algorithm.

Lemma 1 ([10]). (a) Algorithm 2SRP computes an integral solution $(x, y)$ that is a $(1,2,2)$ approximation of the optimal fractional solution $\left(x^{*}, y^{*}\right)$.

(b) Algorithm $1 S R P$, parameterized by $c \in\left(0, \frac{1}{2}\right]$, computes an integral solution $(x, y)$ that is a $\left(\frac{1}{c}, \frac{1}{1-c}, \frac{1}{1-c}\right)$-approximation of the optimal fractional solution $\left(x^{*}, y^{*}\right)$.

The currently best known 1.8-approximation algorithm [10] is obtained by simply running 2SRP with probability $\frac{3}{5}$ and $1 \mathrm{SRP}$ with probability $\frac{2}{5}$, setting $c=\frac{1}{3}$ in the latter.

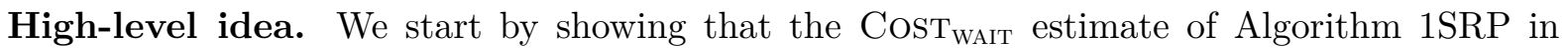
Lemma 1.b is not tight. To analyze it more accurately, we define a shipping pace of an algorithm and show a connection between the shipping pace and the waiting cost. We use that to show that, for $c=\frac{1}{3}$, Algorithm 1SRP computes in fact a $\left(3, \frac{3}{2}, \frac{9}{8}\right)$-approximation. This improvement alone does not reduce the overall approximation ratio of the 1SRP-and-2SRP combination, as it is still dominated by the retailer shipment cost ratio.

However, we will add a third ingredient to this combination: Algorithm LPS, that uses scaling of the fractional solution to obtain a new fractional solution obeying certain deadlines and then applies the recent result on JRP-D, the deadline-constrained variant of JRP [3], to round it to an integral solution. By carefully choosing the scaling factor, probabilities of choosing Algorithms 2SRP, 1SRP and LPS, and fine-tuning the choice of $c$ in Algorithm 1SRP, we eventually reduce the approximation ratio for JRP to about 1.791.

Shipping pace. To measure the waiting cost of an algorithm, we estimate how fast it satisfies each particular order in comparison to how fast these orders are satisfied in $\left(x^{*}, y^{*}\right)$. In $\left(x^{*}, y^{*}\right)$, the orders can be thought of as being satisfied gradually with time. In particular, a fraction $\sum_{t \in\left[a, t^{\prime}\right] \cap T} y_{\pi, t}^{*}$ of an order $\pi=(\rho, a)$ is satisfied till time $t^{\prime}$ (inclusively). For any $\alpha \in[0,1)$, let

$$
\mathrm{ft}_{\mathrm{LP}}(\pi, \alpha)=\min \left\{t \in T: t \geq a \text { and } \sum_{t^{\prime} \geq t} y_{\pi, t^{\prime}}^{*} \leq 1-\alpha\right\}
$$


In other words, $\mathrm{ft}_{\mathrm{LP}}(\pi, \alpha)$ is the first time when the yet un-satisfied fraction of $\pi$ in $\left(x^{*}, y^{*}\right)$ is at most $1-\alpha$.

Let $G:[0,1] \rightarrow \mathbb{R}_{\geq 0}$ be an integrable function such that $\int_{0}^{1} G(z) \mathrm{d} z=1$. We say that a (randomized) algorithm $\mathcal{A}$ has a shipping pace $G$ if for any order $\pi=(\rho, a) \in \Pi$ and $\alpha \in[0,1)$, it holds that

$$
\operatorname{Pr}\left[\mathcal{A} \text { ships at time } t \in\left[a, \mathrm{ft}_{\mathrm{LP}}(\pi, \alpha)\right]\right] \geq \int_{0}^{\alpha} G(z) \mathrm{d} z .
$$

Note that a shipping pace is not unique; it is simply a lower bound on the shipping probability.

Lemma 2. Let $\mathcal{A}$ be a (randomized) algorithm with shipping pace $G$ that produces a solution $(x, y)$. Then,

$$
\operatorname{CosT}_{\text {WAIT }}(x, y) \leq \operatorname{CosT}_{\text {WAIT }}\left(x^{*}, y^{*}\right) \cdot \sup _{w \in[0,1)}\left\{\frac{1}{1-w} \cdot \int_{w}^{1} G(z) \mathrm{d} z\right\} .
$$

Proof. We show that the relation above holds for the waiting cost of any individual order $\pi=$ $(\rho, a, h)$. For the sake of this proof, we number all the consecutive times from set $\{t \in T: t \geq a\}$ as $t_{0}=a, t_{1}, t_{2}, \ldots$. Then the waiting cost associated with $\pi$ is

$$
\operatorname{CosT}_{\mathrm{WAIT}}^{\pi}(x, y)=\sum_{i \geq 0} h\left(t_{i}\right) \cdot \mathbf{E}\left[y_{\pi, t_{i}}\right]=\sum_{i \geq 0}\left[h\left(t_{i+1}\right)-h\left(t_{i}\right)\right] \cdot \sum_{j \geq i+1} \mathbf{E}\left[y_{\pi, t_{j}}\right],
$$

and the waiting cost of $\pi$ in $\left(x^{*}, y^{*}\right)$ can be expressed analogously (but without taking expected values). It is thus sufficient to compare $\sum_{j \geq i+1} \mathbf{E}\left[y_{\pi, t_{j}}\right]$ with $\sum_{j \geq i+1} y_{\pi, t_{j}}^{*}$. Let $\alpha=\sum_{j=0}^{i} y_{\pi, t_{j}}^{*}=$ $1-\sum_{j \geq i+1} y_{\pi, t_{j}}^{*}$. Then, by the definition of (5), $\mathrm{ft}_{\mathrm{LP}}(\pi, \alpha) \leq t_{i+1}$, and therefore

$\sum_{j \geq i+1} \mathbf{E}\left[y_{\pi, t_{j}}\right]=\operatorname{Pr}\left[\mathcal{A}\right.$ ships at time $\left.t>t_{i+1}\right] \leq \operatorname{Pr}\left[\mathcal{A}\right.$ ships at time $\left.t>\mathrm{ft}_{\mathrm{LP}}(\pi, \alpha)\right] \leq \int_{\alpha}^{1} G(z) \mathrm{d} z$,

where we consider only shipments to $\rho$. The equality holds because all $y_{\pi, t_{j}}$ are $0-1$ variables and at most one is non-zero. The inequalities follow from $\mathrm{ft}_{\mathrm{LP}}(\pi, \alpha) \leq t_{i+1}$ and the definition of the shipping pace in (6).

Waiting cost of Algorithm 1SRP. We start with a brief description of Algorithm 1SRP (see Algorithm 2 in [10]). The algorithm is parametrized by $c \in\left[0, \frac{1}{2}\right]$. It first computes the optimal fractional solution $\left(x^{*}, y^{*}\right)$ and then it schedules the shipments, in two phases. In the first phase, it schedules the supplier-to-warehouse shipments. Intuitively, one can visualize this schedule in terms of the "virtual warehouse time", equal to the accumulated fractional shipping value for the warehouse, $X_{t}=\sum_{t^{\prime} \leq t} x_{t^{\prime}}$. The algorithm chooses uniformly a random $\psi \in[0, c]$ and schedules the shipments at virtual warehouse times $\psi, \psi+c, \psi+2 c, \ldots$, which then can be translated into real times. More formally, these shipments are scheduled at (real) times $t$ for which there is $i$ such that $X_{t-1}<\psi+i c \leq X_{t}$. In the second phase, we define tentative shipments from the warehouse to each retailer $\rho$. This is done similarly, by choosing a random $\psi_{\rho} \in[0,1-c]$ and tentatively scheduling these shipments at retailer $\rho$ 's virtual times $\psi_{\rho}, \psi_{\rho}+1-c, \psi_{\rho}+2(1-c), \ldots$ For each tentative shipment of $\rho$, say at a (real) time $t$, the actual shipment to $\rho$ will take place at the first time $t^{\prime} \geq t$ for which there is a supplier-to-warehouse shipment.

Observation 1. Algorithm 1SRP, with parameter $c \in\left(0, \frac{1}{2}\right]$, has a shipping pace

$$
G_{1 \mathrm{SRP}}(z)=\frac{1}{1-c} \cdot \begin{cases}z / c & \text { for } z \in[0, c), \\ 1 & \text { for } z \in[c, 1-c), \\ (1-z) / c & \text { for } z \in[1-c, 1] .\end{cases}
$$


Proof. (Sketch.) Every order is analyzed as if it was waiting first for a shipment (in the computed integral solution) at its retailer and then at the warehouse, with the analysis carried out with respect to the retailer's virtual time (the amount by which the fractional solution satisfies the order). Then the waiting at the retailer has uniform distribution $U[0,1-c]$ and the waiting at the warehouse is upper bounded with a uniform distribution $U[0, c]$. Hence, the distribution of the total waiting time is bounded by a convolution of the two uniform distributions, which results in the trapezoidal shape of the shipping pace $G_{1 \mathrm{SRP}}(x)$, see Figure 1

Side note. We can use Observation 1 along with Lemma 2 to improve the waiting cost ratio of Algorithm 1SRP. Namely, the supremum of $\frac{1}{1-w} \cdot \int_{w}^{1} G_{1 \mathrm{SRP}}(z) \mathrm{d} z$ is achieved for $z=c$ and is then equal to $(2-3 c) /\left(2(1-c)^{2}\right)$. Setting $c=\frac{1}{3}$, we obtain that Algorithm 1SRP returns a $\left(3, \frac{3}{2}, \frac{9}{8}\right)$-approximation. As we noted earlier, this result alone cannot improve the combination of Algorithms 2SRP and 1SRP, because we improved only the third coefficient of the ratio.

Algorithm LPS. To improve the overall approximation guarantee, we therefore need to improve the two first coefficients in the approximation ratio. To this end, we design a new algorithm that performs well in terms of warehouse and retailer shipping costs and has a bounded waiting cost ratio. This algorithm (see below) randomly scales up the optimum solution $\left(x^{*}, y^{*}\right)$, then it converts the scaled solution into an instance of JRP-D, the variant of JRP with deadlines, to which it applies an approximation algorithm from [3]. The algorithm uses a probability distribution $D$ of the scaling parameter that we will define later.

\section{Algorithm LPS}

1. Choose $\zeta \in(0,1]$ from a distribution with the density function $D:(0,1] \rightarrow \mathbb{R}_{\geq 0}$.

2. Compute an optimal fractional solution $\left(x^{*}, y^{*}\right)$.

3. Create a new fractional solution $(\widehat{x}, \widehat{y})$ by setting $\widehat{x}=\min \left\{1, x^{*} / \zeta\right\}$, and (greedily) choosing $\widehat{y}$ to minimize the waiting cost, subject to fixed fractional shipments $\widehat{x}$.

4. Create an instance $\mathcal{L}$ of JRP-D, by inserting a deadline for each order $\pi$ at the first time $t^{\prime}$ for which $\sum_{t<t^{\prime}} \hat{y}_{\pi, t} \geq 1$. (Thus in $(\widehat{x}, \widehat{y})$ each order is served "just in time".)

5. Solve instance $\mathcal{L}$ by using the $\lambda$-approximation algorithm from [3], where $\lambda \approx 1.574$, and return the obtained solution.

Lemma 3. Let $\xi=\int_{0}^{1} \frac{1}{z} \cdot D(z) \mathrm{d} z$. Algorithm LPS produces an integral solution $(x, y)$ with

$$
\begin{aligned}
& \operatorname{CosT}_{\mathrm{WSHIP}}(x, y) \leq \lambda \cdot \xi \cdot \operatorname{CosT}_{\mathrm{WSHIP}}\left(x^{*}, y^{*}\right) \text { and } \\
& \operatorname{CosT}_{\mathrm{RSHIP}}(x, y) \leq \lambda \cdot \xi \cdot \operatorname{CoST}_{\mathrm{RSHIP}}\left(x^{*}, y^{*}\right) .
\end{aligned}
$$

Proof. We analyze the output $(x, y)$ of Algorithm LPS for a fixed $\zeta \in(0,1]$. By Step 3, $\operatorname{CosT}_{\mathrm{WSHIP}}(\widehat{x}, \widehat{y}) \leq(1 / \zeta) \cdot \operatorname{CosT}_{\mathrm{WSHIP}}\left(x^{*}, y^{*}\right)$ and by $\operatorname{Step} 5, \operatorname{CosT}_{\mathrm{WSHIP}}(x, y) \leq \lambda \cdot \operatorname{CosT}_{\mathrm{WSHIP}}(\widehat{x}, \widehat{y})$. Thus, $\operatorname{CosT}_{\mathrm{WSHIP}}(x, y) \leq(\lambda / \zeta) \cdot \operatorname{CosT}_{\mathrm{WSHIP}}\left(x^{*}, y^{*}\right)$. By integrating the estimate above over the probability distribution of $\zeta$, we immediately obtain the first property of the lemma. The proof for the second property is analogous.

Lemma 4. Fix any $\zeta \in(0,1]$ and let $(x, y)$ be the solution returned by Algorithm LPS for this fixed $\zeta$. Fix also an order $\pi=(\rho, a, h) \in \Pi$. Let $\operatorname{shp}(\pi) \geq a$ be the time of the shipment that satisfies $\pi$ in $(x, y)$, that is $y_{\pi, \operatorname{shp}(\pi)}=1$ and $y_{\pi, t}=0$ for $t \neq \operatorname{shp}(\pi)$. Then $\sum_{t \geq \operatorname{shp}(\pi)} y_{\pi, t}^{*} \geq 1-\zeta$.

Proof. The solution $(x, y)$ obeys the deadlines of instance $\mathcal{L}$. The deadlines are set exactly to satisfy the bound of the lemma, i.e., for each order $\pi$ at least $1-\zeta$ fraction of $\pi$ is still to be sent in $\left(x^{*}, y^{*}\right)$ at the time of the deadline inserted for $\pi$.

Observation 2. Algorithm LPS has a shipping pace $G_{\mathrm{LPS}} \equiv D$. 


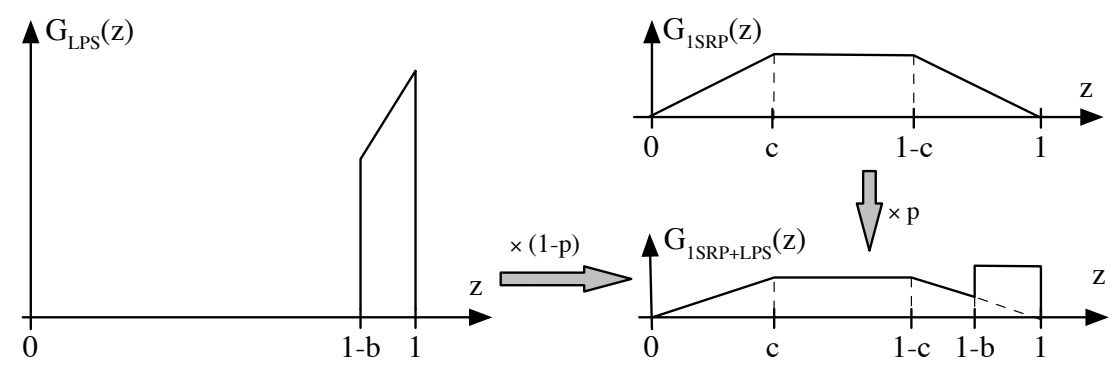

Figure 1: Shipping paces of Algorithms 1SRP, LPS and 1SRP+LPS.

Proof. By Lemma 4, for any $\pi$ and $\zeta$, Algorithm LPS plans a shipment for $\pi$ no later than when a fraction $\zeta$ of $\pi$ is satisfied by $\left(x^{*}, y^{*}\right)$. Thus $D$ is indeed a shipping pace of the algorithm.

Combining Algorithms 1SRP and LPS. Algorithm 1SRP+LPS simply runs Algorithm 1SRP with probability $p$ and Algorithm LPS with probability $1-p$. (Recall that we still have to choose parameter $c$ in Algorithm 1SRP and the probability density $D$ in Algorithm LPS.) We observe that such an algorithm has pace $G_{1 \mathrm{SRP}+\mathrm{LPS}} \equiv p \cdot G_{1 \mathrm{SRP}}+(1-p) \cdot G_{\mathrm{LPS}}$. The following result is an immediate consequence of (i) using Lemma 1.b and Lemma 3 to estimate the total warehouse and retailer shipping costs, (ii) applying Lemma 2 and Observation 2 to estimate the waiting costs, and (iii) using the inequality $\sup _{w \in[0,1]}\left(\int_{w}^{1} G(z) \mathrm{d} z\right) /(1-w) \leq \sup _{z \in[0,1]} G(z)$.

Lemma 5. Let $\xi=\int_{0}^{1} \frac{1}{z} \cdot D(z) \mathrm{d} z$. Algorithm $1 S R P+L P S$ computes an integral solution $(x, y)$ that is a $\left(r_{1}, r_{2}, r_{3}\right)$-approximation of the optimal fractional solution $\left(x^{*}, y^{*}\right)$, where

$$
r_{1}=p / c+(1-p) \lambda \xi, \quad r_{2}=p /(1-c)+(1-p) \lambda \xi, \quad \text { and } \quad r_{3}=\sup _{z \in[0,1]} G_{1 \operatorname{SRP}+\operatorname{LPS}}(z) .
$$

Our next step is to choose a probability density function $D$ in Algorithm LPS. This choice affects the approximation ratio in two ways. On the one hand, we want the value of $\xi$ in Lemma 5 to be as small as possible. To this end, the probability mass should be accumulated close point 1. On the other hand, we need to take into account that Algorithm 1SRP+LPS approximates the waiting cost within the factor $\sup _{z \in[0,1]} G_{1 \mathrm{SRP}+\mathrm{LPS}}(z)$, where $G_{1 \mathrm{SRP}+\mathrm{LPS}} \equiv p \cdot G_{1 \mathrm{SRP}}+(1-$ $p) \cdot G_{\mathrm{LPS}}=p \cdot G_{1 \mathrm{SRP}}+(1-p) \cdot D$.

Therefore, we choose $D$ to be supported on the interval $[1-b, 1]$, where $b \leq c$ is a parameter that we will fix later. Furthermore, we choose $D$ to be such an increasing linear function that the resulting function $G_{1 \mathrm{SRP}+\mathrm{LPS}}$ is constant on $[1-b, 1]$, cf. Figure 1 . To this end, we require that - within the interval $[1-b, 1]$ - the slope of the function $(1-p) \cdot G_{\text {LPS }} \equiv(1-p) \cdot D$ matches the negated slope of the function $p \cdot G_{1 \mathrm{SRP}}$. These considerations imply that once we fix parameters $p, c$ and $b$, the probability density $D$ should be

$$
D(z)=\alpha \cdot z+\frac{1}{b}+\frac{\alpha \cdot b}{2}-\alpha, \quad \text { where } \quad \alpha=\frac{p}{(1-p) \cdot c \cdot(1-c)},
$$

for $z \in[1-b, 1]$ and zero outside of this interval. Straightforward calculations verify that $D$ is indeed a probability density, i.e., $\int_{0}^{1} D(x) \mathrm{d} x=1$. Furthermore, $G_{1 \mathrm{SRP}+\mathrm{LPS}} \equiv p \cdot G_{1 \mathrm{SRP}}+(1-$ $p) \cdot G_{\mathrm{LPS}}$ is constant on the interval $[1-b, 1]$ and its value there is equal to $G_{1 \mathrm{SRP}+\mathrm{LPS}}(1)=$ $(1-p) \cdot D(1)=(1-p) / b+p b /(2 c(1-c))$. Thus

$\xi=\int_{0}^{1} \frac{1}{z} \cdot D(z) \mathrm{d} z=\int_{1-b}^{1} \alpha \mathrm{d} z+\left(\frac{1}{b}+\frac{\alpha \cdot b}{2}-\alpha\right) \int_{1-b}^{1} \frac{1}{z} \mathrm{~d} z=\alpha \cdot b-\left(\frac{1}{b}+\frac{\alpha \cdot b}{2}-\alpha\right) \cdot \ln (1-b)$.

We numerically optimize the parameters $p, c$ and $b$. Specifically, we choose $p=0.822599$, $c=0.342538$ and $b=0.136366$. For those values the maximum of function $G_{1 \mathrm{SRP}+\mathrm{LPS}}$ is achieved in the interval $[1-b, 1]$. and is at most 1.549968. Using the bounds of Lemma 5 . 
we conclude that Algorithm 1SRP+LPS is an $\left(R_{1}, R_{2}, R_{2}\right)$-approximation of $\left(x^{*}, y^{*}\right)$, where $R_{1} \leq 2.700277$ and $R_{2} \leq 1.549968$.

Making ends meet: combining all algorithms. Finally, we combine algorithm 1SRP + LPS with algorithm 2SRP. The resulting algorithm 2SRP+1SRP+LPS uses $2 \mathrm{SRP}$ with probability $\left(R_{1}-R_{2}\right) /\left(R_{1}-R_{2}+1\right)$ and $1 \mathrm{SRP}+\mathrm{LPS}$ with probability $1 /\left(R_{1}-R_{2}+1\right)$. Such algorithm is a $(R, R, R)$-approximation (of the fractional optimal solution), where

$$
R=\frac{2 \cdot R_{1}-R_{2}}{R_{1}-R_{2}+1} \leq 1.790713 .
$$

We therefore obtained the following result.

Theorem 3. There is a polynomial-time 1.791-approximation algorithm for JRP.

\section{A Lower Bound of 2.754 for Online JRP-L}

We now show our lower bound of 2.754 for the competitive ratios for JRP, which improves the previous upper bound of 2.64 by Buchbinder et al. [6]. Since we use only linear waiting-cost functions in our construction, as in [6], our result applies to JRP-L as well.

Single-phase game. In our lower-bound proof it will be convenient to consider a simple version of JRP-L that we refer to as the Single-Phase JRP-L. In the Single-Phase JRP-L all orders are issued at the beginning at time 0 . The waiting cost is assumed to be linear. In addition to the set of retailers and orders, the instance specifies also an expiration time $\theta$. At time $\theta$ all orders expire: they need not be satisfied anymore, but each incurs the waiting $\operatorname{cost} h(\theta)=\theta$. Note that all information about the instance is known to the online algorithm, except for $\theta$, which represents the adversary strategy. Thus the Single-Phase JRP-L is in fact a generalization of the well studied rent-or-buy problem.

We claim that a lower bound of $R$ for Single-Phase JRP-L implies a lower bound of $R$ for JRP-L (and thus for JRP as well). Since a similar argument appeared before in [6, 4], we only briefly sketch the proof of this claim. Suppose that we have an adversary strategy that forces ratio $R$ for Single-Phase JRP-L. We modify it into an adversary strategy that forces the same ratio for JRP-L. This strategy creates a large number of single-phase instances, concatenated together, with the $i$-th instance scaled by a factor of $M^{i}$, for some very large $M$, in the following sense: each order is replaced by $M^{i}$ identical orders and the time is accelerated by a factor of $M^{i}$ as well. By accelerating the time we mean that all time values used to make decisions in the strategy are multiplied by $M^{-i}$. The adversary applies the same strategy in each phase, forcing ratio $R$ for each phase. Each phase may produce some number of non-satisfied orders, but these can be satisfied by one shipment for all retailers at the end of the game. This will add only a constant to the adversary shipment cost and, since the phase lengths are decreasing so fast, the increase of the adversary's waiting cost will be also negligible.

Single-phase construction. We will use an instance of Single-Phase JRP-L with $N+1$ retailers in $\mathcal{R}$, denoted $\rho_{0}, \rho_{1}, \ldots, \rho_{N}$. The costs of shipping from the warehouse to each of them are as follows: $\mathrm{c}_{\rho_{0}}=\mathrm{c}_{0}=0$ and $\mathrm{c}_{\rho_{i}}=\mathrm{c}$ for all $i>0$ and $\mathrm{c}$ that we fix later. These are normalized so that $\mathrm{C}=1$, i.e., the cost of shipping from the supplier to the warehouse is 1 .

Each retailer $\rho_{i}$ places $w_{i}$ identical orders $\left(\rho_{i}, 0, h_{i}\right)$ at time 0 , where $h_{i}(t)=t$ for all $i$. Equivalently, we can view this as issuing a single order $\pi_{i}=\left(\rho_{i}, 0, h_{i}^{\prime}\right)$ with weight $w_{i}$, that is with waiting cost function $h_{i}^{\prime}(t)=w_{i} \cdot t$. We will adapt this terminology in this section. We choose the weights to be quickly decreasing, that is $w_{i} \gg w_{i+1}$ for all $i<N$, so that the slopes of the functions $h_{i}^{\prime}$ are decreasing rapidly with $i$. As a result, in the proof below, when the algorithm satisfies an order $\pi_{i}$, the waiting costs (of the algorithm and the adversary) of all orders $\pi_{i+1}, \pi_{i+2}, \ldots$ will be negligible. For clarity, in the calculations below we will assume 
these costs to be 0 . (By adjusting the weights appropriately, we can make these costs at most an arbitrarily small $\epsilon$, and then our lower bound will approach $R$.)

Let $\mathcal{A}$ be an online algorithm for Single-Phase JRP-L. To describe the adversary strategy, we first normalize the way $\mathcal{A}$ proceeds. Using a simple exchange argument, it is easy to show that, without loss of generality, $\mathcal{A}$ satisfies all the demands in increasing order of their indices, i.e., if $i<j$ then $\pi_{i}$ is satisfied earlier than or together with $\pi_{j}$. Then, the adversary stops the game the moment that $\mathcal{A}$ satisfies more than one order with a single shipment. ("Stopping" means that the expiration time $\theta$ is set to the current time.) To complete the strategy's description we can thus focus on $\mathcal{A}$ satisfying orders $\pi_{0}, \pi_{1}, \ldots$ in this order, each with a dedicated shipment. If the waiting cost associated with $\pi_{i}$ at the moment of its satisfaction is smaller than a certain threshold value $\sigma_{i}$, the game ends, otherwise it continues. This means that as long as the game did not end at of before $\mathcal{A}$ 's shipment satisfying $\pi_{i}, \mathcal{A}$ 's cost for these shipments is at least $\sum_{j=0}^{i}\left(1+\mathrm{c}_{j}+\sigma_{j}\right)$. In particular, if the game does not end due to any aforementioned reason at or before the time that $\mathcal{A}$ satisfies $\pi_{N}$, then the game ends naturally with this shipment; otherwise we say that the game ends prematurely.

As was the case with $\mathrm{c}_{i}$ 's, all the thresholds $\sigma_{i}$ coincide and are denoted $\sigma$, with the exception of $\sigma_{0}$. We now give the values of all the parameters. We let $\mathrm{c}$ be the only real root of

$$
c^{2}(c+1)=1
$$

and

$$
\sigma_{0} \equiv \frac{1}{\mathrm{c}+1}=\mathrm{c}^{2}, \quad \sigma \equiv \sigma_{0}^{2}=\mathrm{c}^{4}=\mathrm{c}^{2}+\mathrm{c}-1,
$$

where the identities follow from $(8)$. We have $\mathrm{c} \approx 0.7548, \sigma_{0} \approx 0.5698$ and $\sigma \approx 0.3247$.

We claim that unless the game ends naturally, the competitive ratio of $\mathcal{A}$ is at least $R=2+\mathrm{c}$. To see this, let us consider all the ways in which the game can end prematurely.

Let $\omega$ be $\mathcal{A}$ 's waiting cost of $\pi_{0}$ when it satisfies $\pi_{0}$. If $\omega<\sigma_{0}$ then $\mathcal{A}$ 's cost is at least $1+\omega$, whereas OPT can pay the waiting cost $\omega$ alone, resulting in ratio no smaller than

$$
1+\frac{1}{\sigma_{0}}=2+\mathrm{c}=R
$$

If $\mathcal{A}$ satisfies $\pi_{0}$ together with another order by a single shipment, then $\mathcal{A}$ 's cost is at least $1+\mathrm{c}+\omega$ whereas OPT will either pay the waiting cost $\omega$ for $\pi_{0}$ or 1 for satisfying $\pi_{0}$ at time 0 . Thus the competitive ratio is at least

$$
\frac{1+\mathrm{c}+\omega}{\min \{1, \omega\}} \geq \frac{2+\mathrm{c}}{1}=2+\mathrm{c}=R .
$$

Now we consider analogous two cases regarding the shipment for $\pi_{i}$, where $i \geq 1$, assuming that the game did not end before. This means that $\mathcal{A}$ already suffered a cost of at least $\sigma_{0}+1+(i-1)(\sigma+1+\mathrm{c})$ associated with satisfying orders $\pi_{0}, \ldots, \pi_{i-1}$, plus some additional cost associated with satisfying $\pi_{i}$. Let now $\omega$ denote the waiting cost of $\pi_{i}$ when $\mathcal{A}$ satisfies $\pi_{i}$.

If $\omega<\sigma$ then OPT satisfies the orders $\pi_{j}$ for all $j<i$ with a single shipment at time 0 , and pays the waiting cost $\omega$ for $\pi_{i}$. The competitive ratio is at least

$$
\frac{\sigma_{0}+1+(i-1)(\sigma+1+\mathrm{c})+\omega+1+\mathrm{c}}{1+(i-1) \mathrm{c}+\omega}=1+\frac{i+\mathrm{c}+\sigma_{0}+(i-1) \sigma}{1+(i-1) \mathrm{c}+\omega} \geq 1+\frac{i+\mathrm{c}+\sigma_{0}+(i-1) \sigma}{1+(i-1) \mathrm{c}+\sigma},
$$

which after substituting formulas (9) for $\sigma_{0}$ and $\sigma$, as well as using (8), becomes

$$
1+\frac{1+i \mathrm{c}+i \mathrm{c}^{2}}{i \mathrm{c}+\mathrm{c}^{2}}=2+\frac{1+i \mathrm{c}^{2}-\mathrm{c}^{2}}{\mathrm{c}(i+\mathrm{c})}=2+\frac{i \mathrm{c}^{2}+\mathrm{c}^{3}}{\mathrm{c}(i+\mathrm{c})}=2+\mathrm{c}=R
$$

Let us consider the remaining case in which $\mathcal{A}$ satisfies another order together with $\pi_{i}$. In this case Opt satisfies all the previous orders with a single shipment at time 0 ; as for $\pi_{i}$, Opt 
either satisfies it with that shipment as well, or pays the waiting cost $\omega$ for $\pi_{i}$, whichever is cheaper. Thus the ratio is at least

$$
\frac{\sigma_{0}+1+(i-1)(\sigma+1+\mathrm{c})+\omega+1+2 \mathrm{c}}{1+(i-1) \mathrm{c}+\min \{\mathrm{c}, \omega\}} \geq 1+\frac{i+2 \mathrm{c}+\sigma_{0}+(i-1) \sigma}{1+i \mathrm{c}},
$$

which after substituting formulas (9) for $\sigma_{0}$ and $\sigma$, becomes

$$
1+\frac{1+(i+1) \mathrm{c}+i \mathrm{c}^{2}}{1+i \mathrm{c}}=1+\frac{(1+\mathrm{c})(1+i \mathrm{c})}{1+i \mathrm{c}}=2+\mathrm{c}=R .
$$

Thus the ratio is at least $R$ if the game ends prematurely. But if it does not, then $\mathcal{A}$ 's cost for each shipment, except the one for $\pi_{0}$, is at least $1+c+\sigma=c^{2}+2 c$, by (9). On the other hand, OPT satisfies all orders with a single shipment at time 0 , which costs $1+N$ c. With $N \rightarrow \infty$, OpT's cost of 1 for shipment from the supplier to the warehouse becomes negligible and OpT's cost per order tends to c. Therefore, the competitive ratio tends to $R=2+\mathrm{c}$. Summarizing the above argument, we obtain:

Theorem 4. Each online deterministic algorithm for JRP-L has competitive ratio at least 2.754 .

\section{Tight Bound of 2 for Online JRP-D}

We now present an online algorithm for JRP-D with competitive ratio 2, matching the lower bound that is given in Appendix A. We will denote the shipments of the algorithm by $\left(B_{1}, t_{1}\right)$, $\left(B_{2}, t_{2}\right), \ldots$, where $t_{1} \leq t_{2} \leq \ldots$. The set $B_{j}$ of retailers participating in the $j$-th shipment is called the $j$ th batch. For convenience, we introduce a "dummy" 0 'th shipment at time $t_{0}=0$, which we think of as if it shipped to all the retailers in the instance at no cost. (All that matters is that at time 0 each retailer does not have any pending orders.) For a retailer $\rho$ and time $t$, we define the deadline of $\rho$ to be the earliest deadline of a pending order in $\rho$. If a retailer does not have any pending orders, its deadline is $+\infty$. Without loss of generality, we can assume that all shipments (of an online algorithm and the adversary) take place only at deadlines of some retailers. If $t_{j}$ is the deadline of a retailer $\rho$ then we say that $\rho$, or the order in $\rho$ with deadline $t_{j}$, triggers shipment $\left(B_{j}, t_{j}\right)$.

Algorithm $\mathcal{G}$ : Suppose that we just completed shipment $\left(B_{j-1}, t_{j-1}\right)$. We wait until we reach a deadline of a retailer, which will become the trigger retailer for the $j$ th shipment. We denote this retailer by $\chi_{j}$ and its deadline by $t_{j}$. At time $t_{j}$ our batch is $B_{j}=\left\{\chi_{j}\right\} \cup X_{j}$, where $X_{j}$ contains the maximum number of retailers, in order of increasing deadlines, such that $\mathrm{c}\left(X_{j}\right) \leq \mathrm{C}$.

If $j=1$ then, according to our convention, $j-1=0$ refers to the dummy shipment at time $t_{0}=0$. Thus the first shipment will occur at the first deadline of the instance.

Analysis. We now analyze this algorithm. To simplify the analysis we will assume that all order arrival times and deadlines are different. The instance can be converted to have this property by an infinitesimal perturbation of arrival times and deadlines.

We divide the sequence of shipments into phases. A phase is a maximal interval $[g, h]$ of integers (indices of shipments), where $1 \leq g \leq h$, such that the adversary does not make any shipments in the time interval $\left(t_{g}, t_{h}\right]$. In other words: (i) there are no adversary shipments in $\left(t_{g}, t_{h}\right]$, (ii) the adversary shipped in $\left(t_{g-1}, t_{g}\right]$, and (iii) either $t_{h}$ is the last deadline or the adversary shipped in $\left(t_{h}, t_{h+1}\right]$. Note that the first phase starts with the first shipment (that is $g=1)$. Indeed, the adversary must ship in the interval $\left(t_{0}, t_{1}\right]$, because $t_{1}$ is the first deadline. The lemma below elucidates a property of phases that will be critical to our analysis.

Lemma 6. Let $[g, h]$ be a phase and $g<j \leq h$. Let $\pi$ be the order in $\chi_{j}$ that triggers shipment $B_{j}$. Then $\pi$ was pending at time $t_{j-1}$, and among all orders pending at time $t_{j-1}$ it was the earliest-deadline order not in $B_{j-1}$. 
Proof. Suppose that $\pi=\left(\chi_{j}, a, d\right)$, that is $d=t_{j}$. If we had $a>t_{j-1}$ then the adversary would have to make a shipment in the interval $(a, d]$, but this would contradict the definition of a phase. (Recall that, by our assumptions, the adversary cannot ship at time $a$.) So $\pi$ was pending at time $t_{j-1}$. It must also be in fact the earliest-deadline order outside $B_{j-1}$, because $B_{j}$ is the first shipment after $B_{j-1}$.

Lemma 7. Let $[g, h]$ be a phase and $g<j \leq h$. Suppose that $\rho \in B_{j}$, where for $j=h$ we assume that $\rho=\chi_{h}$. Let $j^{\prime}<j$ be maximum such that $\rho \in B_{j^{\prime}}$ (if there is no shipment to $\rho$ before $t_{j}$, let $\left.j^{\prime}=0\right)$. Then the adversary must ship to $\rho$ in the interval $\left(t_{j^{\prime}}, t_{h}\right]$, whence $j^{\prime}<g$.

Proof. Right after the shipment at time $t_{j^{\prime}}$, there were no orders in $\rho$, so there must be an order that arrived after $t_{j^{\prime}}$ and has deadline at most $t_{h}$, by the algorithm, and by the fact that $\rho \notin X_{h}$. So the adversary must ship in the time interval $\left(t_{j^{\prime}}, t_{h}\right]$. This in turn implies that $j^{\prime}<g$, by the definition of phases.

Consider a phase $[g, h]$. Using the above lemma, if $\rho \in B_{j}$, where either $g \leq j<h$ or $j=h$ and $\rho=\chi_{h}$, then with the $\mathcal{G}$ 's warehouse-to- $\rho$ shipment at time $t_{j}$ we can associate a unique warehouse-to- $\rho$ shipment of the adversary that occurred not later than at time $t_{h}$. With this in mind, we can now analyze Algorithm $\mathcal{G}$ using a charging argument, as follows:

- We charge $c\left(\chi_{g}\right)$, namely the cost of the warehouse-to- $\chi_{g}$ shipment at time $t_{g}$ to the associated warehouse-to- $\chi_{g}$ shipment of the adversary (as described above). The charging ratio here is 1 .

- We charge $C+c\left(X_{h}\right)$, representing the cost of the first supplier-to-warehouse shipment at time $t_{g}$ and the cost of the warehouse-to- $X_{h}$ shipment at time $t_{h}$, to the adversary cost of $\mathrm{C}$ of the supplier-to-warehouse shipment cost right before $t_{g}$. Since $\mathrm{c}\left(X_{h}\right) \leq \mathrm{C}$, the charging ratio is at most 2 .

- For $j=g, \ldots, h-1$, we charge the cost $\mathrm{C}+\mathrm{c}\left(X_{j}\right)+\mathrm{c}\left(\chi_{j+1}\right)$, that represents the supplierto-warehouse shipment cost at time $t_{j+1}$ and the cost of shipments warehouse-to- $X_{j}$ and warehouse-to- $\chi_{j+1}$, to $\mathrm{c}\left(X_{j}\right)+\mathrm{c}\left(\chi_{j+1}\right)$, namely the adversary's warehouse-to-retailer shipment cost associated with the retailers in $X_{j} \cup\left\{\chi_{j+1}\right\}$. By the choice of $X_{j}$ and Lemma 6 . we have $\mathrm{c}\left(X_{j}\right)+\mathrm{c}\left(\chi_{j+1}\right)>\mathrm{C}$, so the charging ratio is at most 2 .

In all cases the charging ratio is at most 2 , and different charges are assigned to different portions of the adversary cost. Thus Algorithm $\mathcal{G}$ is 2-competitive. In Appendix A we show a matching lower bound.

Theorem 5. (a) Algorithm $\mathcal{G}$ is 2-competitive for JRP-D. (b) Every deterministic online algorithm for JRP-D has competitive ratio at least 2 .

\section{Final Comments}

There are still significant gaps between the lower and upper bounds for the approximability of different variants of JRP. For JRP-D, we have $\mathbb{A P X}$-hardness [12, 3] and an integrality gap of 1.245 [3], while the best upper bound is 1.574 [3]. The case of JRP-L (linear waiting costs), although most natural, is poorly understood. The best upper bound is the same as for the general case, namely 1.791, shown in this paper, even though at this time not even an approximation scheme has been ruled out. Some progress on this problem was recently reported in [13. The approximability of the online version of JRP-L also remains open.

JRP can be naturally generalized to trees of arbitrary depth. This multi-level JRP problem with deadlines was studied by Bechetti et al. 2, who provided a 2-approximation algorithm. Khanna et al. [7] considered the case of linear waiting costs. Very recently, Chaves (private communication) has shown that the general case can be reduced to the so-called multi-stage assembly problem, for which a 2-approximation algorithm was given by Levy et al. 9]. 


\section{References}

[1] Esther Arkin, Dev Joneja, and Robin Roundy. Computational complexity of uncapacitated multi-echelon production planning problems. Operations Research Letters, 8(2):61$66,1989$.

[2] Luca Becchetti, Alberto Marchetti-Spaccamela, Andrea Vitaletti, Peter Korteweg, Martin Skutella, and Leen Stougie. Latency-constrained aggregation in sensor networks. ACM Transactions on Algorithms, 6(1):13:1-13:20, 2009.

[3] Marcin Bienkowski, Jaroslaw Byrka, Marek Chrobak, Neil Dobbs, Tomasz Nowicki, Maxim Sviridenko, Grzegorz Świrszcz, and Neal E. Young. Approximation algorithms for the joint replenishment problem with deadlines. In Proc. of the 40th Int. Colloq. on Automata, Languages and Programming (ICALP), 2013. To appear.

[4] Marcin Bienkowski, Jaroslaw Byrka, Marek Chrobak, Łukasz Jeż, and Grzegorz Stachowiak. Online control message aggregation in chain networks. In Proceedings of WADS'13, 2013. To appear.

[5] Carlos Brito, Elias Koutsoupias, and Shailesh Vaya. Competitive analysis of organization networks or multicast acknowledgement: How much to wait? Algorithmica, 64(4):584-605, 2012 .

[6] Niv Buchbinder, Tracy Kimbrel, Retsef Levi, Konstantin Makarychev, and Maxim Sviridenko. Online make-to-order joint replenishment model: primal dual competitive algorithms. In Proc. of the 19th ACM-SIAM Symp. on Discrete Algorithms (SODA), pages 952-961, 2008.

[7] Sanjeev Khanna, Joseph Naor, and Danny Raz. Control message aggregation in group communication protocols. In Proc. of the 29th Int. Colloq. on Automata, Languages and Programming (ICALP), pages 135-146, 2002.

[8] Retsef Levi, Robin Roundy, and David B. Shmoys. A constant approximation algorithm for the one-warehouse multi-retailer problem. In Proc. of the 16th ACM-SIAM Symp. on Discrete Algorithms (SODA), pages 365-374, 2005.

[9] Retsef Levi, Robin Roundy, and David B. Shmoys. Primal-dual algorithms for deterministic inventory problems. Mathematics of Operations Research, 31(2):267-284, 2006.

[10] Retsef Levi, Robin Roundy, David B. Shmoys, and Maxim Sviridenko. A constant approximation algorithm for the one-warehouse multiretailer problem. Management Science, 54(4):763-776, 2008.

[11] Retsef Levi and Maxim Sviridenko. Improved approximation algorithm for the onewarehouse multi-retailer problem. In Proc. of the 9th Int. Workshop on Approximation Algorithms for Combinatorial Optimization (APPROX), pages 188-199, 2006.

[12] Tim Nonner and Alexander Souza. Approximating the joint replenishment problem with deadlines. Discrete Mathematics, Algorithms and Applications, 1(2):153-174, 2009.

[13] Tim Nonner and Maxim Sviridenko. An efficient polynomial-time approximation scheme for the joint replenishment problem. In Proc. Integer Programming and Combinatorial Optimization - 16th International Conference (IPCO'13), pages 314-323, 2013. 


\section{A A Lower Bound of 2 for Online JRP-D}

In this section we show that no online algorithm for JRP-D can have competitive ratio smaller than 2, thus proving Theorem 5(b). Similar to the proof in Section 4, we actually provide this lower bound for the restricted variant of JRP-D called Single-Phase JRP-D. In SinglePhase JRP-D, all orders arrive at time 0 . The adversary can stop the game at any time $\theta$ (the expiration time), unknown to the online algorithm. All orders not satisfied by time $\theta$ incur no cost. By an argument similar to the one given in Section 4, any lower bound for Single-Phase JRP-D implies the same lower bound for JRP-D. (For JRP-D the argument in Section 4 has to be slightly refined; the details will be given in the final version of this paper.)

In our instance, the supplier-to-warehouse shipping cost is $\mathrm{C}=1$. We have $N+1$ retailers $\rho_{i}, i=0,1, \ldots, N$, for some sufficiently large $N$. Retailer $\rho_{0}$ has shipping cost $\mathrm{c}_{\rho_{0}}=0$, and each retailer $\rho_{i}$, for $i>0$, has shipping cost $\mathrm{c}_{\rho_{i}}=1$. For each $i$, retailer $\rho_{i}$ issues one order $\pi_{i}$ at time 0 with waiting cost function

$$
h_{i}(t)= \begin{cases}0 & 0 \leq t \leq i \\ \infty & t>i\end{cases}
$$

Let $\mathcal{A}$ be an online algorithm for JRP-D. $\mathcal{A}$ must ship to each $\rho_{i}$ no later than at time $i$. Without loss of generality, $\mathcal{A}$ ships only at integer times, so as long as $\mathcal{A}$ ships to each retailer separately then each $\rho_{i}$ will be shipped at time $i$. The adversary will stop the game as soon as $\mathcal{A}$ ships to more than one retailer. If this does not happen, the game stops after the shipments to all retailers, that is right after time $N$.

We argue now that this forces the competitive ratio of $\mathcal{A}$ to be arbitrarily close to 2 . If $\mathcal{A}$ ships to each retailer separately, it pays 1 for retailer 0 and 2 for each retailer $\rho_{i}, i \geq 1$, for the total cost of $2 N+1$. The adversary can ship to all retailers at the beginning, paying $N+1$. So the ratio approaches 2 with $N \rightarrow \infty$.

Suppose that at some time $k, \mathcal{A}$ ships to $\rho_{k}$ and some other retailer, and let $k$ be the first such $k$. Then $\mathcal{A}$ 's cost is $1+2(k-1)+3=2 k+2$. The adversary can ship to all retailers $\rho_{0}, \ldots, \rho_{k}$ at the beginning, paying $k+1$. So the ratio is 2 . 